\title{
La construcción de la referencia en torno al concepto de brecha digital en España
}

The construction of the reference around

the concept of digital divide in Spain

A construção da referência em torno do conceito de fenda digital na Espanha

doi:Io.III44/Javeriana.SyP33-64.crbd

Recibido: Agosto I3, 2013 Aceptado: Diciembre 13, 2013

Submission date: August 13, 2013 Acceptance date: December I3, 2013

\section{Origen del artículo}

Este trabajo investigativo se realizó para optar al título de Doctorado en Comunicación, Cambio Social y Desarrollo que imparte el Departamento de Sociología IV de la Facultad de Ciencias de la Información de la Universidad Complutense de Madrid. El trabajo investigativo de tesis doctoral se realizó en calidad de estudiante, no hizo parte de una investigación derivada de un grupo formal de investigación, ni contó con el soporte de entidades financiadoras. Fecha de inicio y finalización de la investigación: junio 2007 a septiembre 20II.

\section{María Cristina Pinto Arboleda}

Comunicadora Social colombiana egresada de la Universidad Externado de Colombia. Doctora Cum Laude en Ciencias de la Información de la Universidad Complutense de Madrid. Experta en Información Internacional y Países del Sur de la misma Universidad. Actualmente se desempeña como profesora e investigadora de la Facultad de Comunicación de la Universidad de Medellín. Especializada en temas de comunicación para el cambio social, ha desarrollado sus actividades profesionales principalmente en UNICEF (España y Nepal). Con experiencia investigativa en los campos de la sociología de la juventud y en nuevas tecnologías de la información y la comunicación. Correspondencia: Circular $\mathrm{I}^{\mathrm{a}}$ No. 70-oI, Medellín, Bloque 7. Campus Laureles. Correo electrónico: mcpintoa@gmail.com 


\section{Resumen}

En el presente artículo se describen los fundamentos teóricos, la ruta metodológica y los principales hallazgos de un trabajo de investigación doctoral que desde el Análisis crítico del discurso, la Teoría de la mediación social y la Economía política de la comunicación, se plantea como objetivo analizar el mapa conceptual y referencial con el que se está elaborando un concepto que ha aparecido en los últimos años para definir un fenómeno amplio y a la vez ambiguo como lo es el de brecha digital; el cual a su vez, hace parte de hilos discursivos relacionados con la inserción de las TIC, Tecnologías de la Información y la Comunicación.

La identificación del conjunto de estructuras lingüísticas presentes en los discursos analizados se ha centrado en el reconocimiento de los sistemas de saberes que se dejan entrever en las distintas expresiones que se ponen de manifiesto sobre los fenómenos sociales.

Palabras Clave: Brecha digital, sociedad de la información, sociedades del conocimiento, análisis crítico del discurso, tecnología de la información y la comunicación TIC.

Descriptores: Brecha digital, sociedad de la información, sociedad del conocimiento, análisis del discurso, tecnologías de la información y la comunicación.

\section{Abstract}

Based on a critical discourse analysis, the theory of social mediation and political economy of communication, the theoretical foundations, methodological route and the main findings of a doctoral research work are described in this article. The objective is to analyze the conceptual and referential map with which a concept is being developed and has appeared in recent years to define a broader phenomenon, yet ambiguous, such as the digital divide; which is also part of discursive threads related to the inclusion of ICT, Information and Communication Technologies. The identification of the linguistic set of structures found in the discourses analyzed has been focused on the recognition of systems of knowledge that can be seen in the various expressions about social phenomena.

Key words: digital divide, information society, knowledge societies, critical discourse analysis, information and communications technology ICT.

Keywords plus: digital divide, information society, knowledge society, discourse analysis, information and communications technologies.

\section{Resumo}

Neste artigo descrevem-se os fundamentos teóricos, a rota metodológica e os principais achados de um trabalho de pesquisa de doutorado que, desde a Análise crítica do discurso, a Teoria da mediação social e a Economia política da comunicação, levanta como objetivo analisar o mapa conceitual e referencial com que está sendo elaborado um conceito que apareceu nos últimos anos para definir um fenômeno amplo, embora ambíguo, como é o de fenda digital; o que, por sua vez, faz parte de fios discursivos relacionados com a inserção das TIC, Tecnologias da Informação e a Comunicação. A identificação do conjunto de estruturas linguísticas presentes nos discursos analisados tem-se centrado no reconhecimento dos sistemas de saberes que deixam entrever nas distintas expressões postas de manifesto sobre os fenômenos sociais.

Palavras-chave: Fenda digital, sociedade da informação, sociedades do conhecimento, análise crítica do discurso, tecnologia da informação e a comunicação TIC.

Descritores: Inclusão digital, sociedade da informação, sociedade do conhecimento, análise do discurso, tecnologia da informação e comunicação. 


\section{La construcción de la referencia en torno al concepto de brecha digital en España}

\section{Introducción}

Los hilos discursivos con los que se construye la referencia ${ }^{1}$ en torno a la adaptación del cambio propiciado por las Tecnologías de la Información y la Comunicación (TIC), cumple un papel estratégico al momento de legitimar la operabilidad y aceptación social de la implementación del cambio tecnológico. Las cuestiones conceptuales y referenciales son determinantes para la construcción de sentido, ya que desde la reproducción de los marcos cognitivos -que son los que operan sobre los relatos difundidos dentro de la comunicación pública para generar modelos de representación del mundo-se configura el orden social que determina el tipo de modelo que se está privilegiando con la inserción de las herramientas tecnológicas.

En el presente artículo se formula como hipótesis de partida, que el discurso promovido desde los documentos institucionales -donde se han definidoconceptos comoel de brecha digital-, ha sido determinante para entender la forma en la que se han legitimado determinadas relaciones de poder dentro de las políticas que incluyen temas referidos a la introducción de las TIC.

El objetivo del trabajo investigativo realizado consistió en analizar el mapa conceptual y referencial con el que se está elaborando un concepto como el de brecha digital. Para ello fue necesario analizar algunos de los documentos institucionales que han concebido este tipo de conceptos, para entender la forma en la que se han legitimado determinadas relaciones de poder a través de la tematización de puntos de debate centrales con los que se han definido las políticas globales de la introducción de las TIC. Así como también identificar la manera en que se ha materializado dicho discurso dentro del debate de la prensa nacional española.

Teniendo en cuenta que el análisis crítico del discurso (ACD) permite identificar aquellas estrategias lingüísticas que contribuyen a reforzar imaginarios y representaciones sociales, donde se promete igualdad y democracia por el uso de las nuevas tecnologías, se consideró utilizar su metodología como una herramienta para comprender cómo se construyen y presentan a la opinión pública, campos cognitivos que conforman el universo temático relacionado con las categorías propuestas de sociedad de la información y sociedades del conocimiento.

Además, esta referencia metodológica se complementó con una propuesta de marco teórico y metodológico planteada desde campos de estudio tales como la Teoría de la mediación social y la Economía política de la comunicación. De esta manera, se desarrolló un trabajo de triangulación teórica pertinente para analizar el discurso desde 
sus componentes ideológicos y cognitivos, y desde la producción misma de los documentos. El principal objetivo buscado con este proceso de triangulación teórica era incrementar la validez de los resultados, contando con un enfoque multidisciplinar interesante para enriquecer el análisis de los resultados obtenidos.

Concretamente con el ACD se pretendía identificar las opciones seleccionadas a nivel discursivo para justificar desde el punto de vista teórico, por qué determinadas interpretaciones de acontecimientos discursivos parecen más válidas que otras. Por su parte, desde la Teoría social de la comunicación se analizó la transformación de la comunicación pública y el cambio de la sociedad, bajo el paradigma de la Teoría de la mediación. Y por último, desde el aporte de la Economía política de la comunicación se interpretaron los principales resultados, bajo la lógica de intervenciones económicas que determinan el acceso a los bienes y servicios info-comunicacionales.

$\mathrm{El}$ aporte de estas disciplinas fue determinante para definir algunas consideraciones relevantes en torno a cuestiones como: las relaciones de poder que contribuyen a dar forma al discurso, los elementos de carácter ideológico, la ubicación del discurso sobre el debate social respecto al tema, y su grado de contribución para mantener las actua- les relaciones sociales. Para esto, se desarrolló una metodología pertinente -la cual se explicará en un apartado posterior-, para analizar qué diferencias estructurales y conceptuales de los discursos contribuyen o no a reforzar determinadas referencias acerca del marco axiológico que conforma el paradigma de la sociedad de la información y el de sociedades del conocimiento, en los que se inserta el concepto de brecha digital.

\section{Marco de referencia}

El reto de introducir las TIC como una herramienta de participación social, para algunos autores como Francisco Sierra y Víctor Manuel Marí (2008), se plantea sobre todo como un problema esencialmente epistemológico, más que de método, argumentando que es necesario reflexionar acerca de las cuestiones conceptuales con las que se trata un tema trascendental como puede ser el del derecho a la comunicación.

Es precisamente sobre los marcos cognitivos de reflexividad e imaginación política analizados por Sierra, que el análisis del discurso permite identificar las dimensiones que desde el orden social se configuran para construir la referencia acerca del modelo de sociedad que se está privilegiando con la inserción de las TIC. Siempre teniendo presente 
como lo expresa Teun Van Dijk (200o, p. I9), que la relación entre discurso y sociedad no es directa sino mediada por la cognición compartida de los miembros sociales (conocimientos, ideologías, normas y valores).

El discurso promocional y publicitario ha encontrado espacio dentro del escenario desenfrenado de la revolución informática (Mattelart, I998, p.3I). Este es propio de los medios de comunicación masiva que a su vez comparten relaciones de intertextualidad con una serie de documentos institucionales elaborados por organizaciones que, a nivel supranacional, establecen las directrices respecto al modelo que cada país debe seguir para adaptarse al cambio tecnológico. Como es sabido, la naturaleza de la producción de los medios de comunicación masiva (MCM) se caracteriza por la necesidad de informar rápidamente acerca de realidades que varían de forma vertiginosa y que por eso mismo, necesitan ser ubicadas dentro de un contexto que explique sus múltiples dimensiones.

\section{Brecha digital: Desigualdades en distribución, aplicación y uso de tecnologías}

Aunque en las fuentes bibliográficas consultadas se citan diferentes fechas referentes al año en que empieza a ser utilizado el término brecha digital, la información coincide en que fue en Estados Unidos donde se acuñó dicha expresión a mediados de la década de I99o, aunque ya desde mediados de I980 se registraron las primeras discusiones públicas acerca del acceso desigual que se empezó a presentar con la proliferación de los primeros ordenadores.

Paul J., citado en Vega-Almeida (2007) identifica la presencia del término en el reporte del Departamento de Comercio de 1995, titulado The digital divide, a survey of the have nots in rural and urban America, año en que también es presentada por el vicepresidente Al Gore la agenda política acerca de la Infraestructura Nacional de la Información. Más adelante en 1998 y 1999 se lanzan los reportes de la Administración Nacional de Telecomunicaciones e Información (NTIA) y el
Departamento de Comercio, denominados New data on the digital divide y Defining the digital divide.

La repercusión del problema en el contexto internacional se registró a partir de la década de I980 con la presentación del informe de la UIT El eslabón perdido en el que la organización describía el acceso desigual entre países a las nuevas tecnologías de la información y dejaba de manifiesto una brecha de acceso al modelo económico emergente que se desarrolló con la inserción de las computadoras en los países desarrollados. Esta preocupación se hace manifiesta por parte del Comité de Naciones Unidas a finales de la década de I990, afirmando que se estaba propiciando una brecha informacional y tecnológica entre países, la cual estaba originando una pobreza de tipo informacional (Vega-Almeida, 2007).

En el contexto internacional el término tomó un significado diferente, fruto del conjunto de desigualdades en la distribución de la riqueza y de los indicadores de desarrollo humano que originaba una segmentación más, entre "info ricos" e "info pobres" (Felicie, 2006). De esta manera se empezaron a analizar las asimetrías de conectividad que se observaban entre las distintas regiones del mundo (por ejemplo, países desarrollados vs. países en vías de desarrollo), también contemplando las diferencias en el acceso a las nuevas TIC que tenían lugar dentro de los mismos países (segmentaciones de acuerdo al estrato socioeconómico, la etnia, el género, la pertenencia ecológica, la edad, etc.).

Años más tarde, Jesús Martín Barbero afirmaba en una conferencia ofrecida en la Universidad Complutense de Madrid que "Se nos rompió la brecha cuando menos lo pensábamos"2; es decir, en pocos años se ha introducido un concepto que no para de tomar nuevas formas y significaciones, según el contexto en el que se analice. Si en un primer momento el problema se reducía a la dotación tecnológica, ahora tiene que ver más con los usos y con el tipo de apropiación social, según las demandas del escenario donde sea analizado. 


\section{Fases / Estados de la Brecha digital}

Jan A.G.M. van Dijk (2005, p. 2I) establece una división conceptual entre cuatro términos específicos relacionados con el acceso a la tecnología digital:

I). Acceso a la motivación, (motivación para usar la tecnología digital).

2). Acceso físico o material (posesión de ordenadores y de conexiones a internet o permiso para usar los dispositivos y sus contenidos).

3). Acceso a las competencias (tener competencias digitales: operativas, informativas y estratégicas).

4). Acceso para el uso (número y diversidad de aplicaciones).

Para el autor una característica apropiada para describir la brecha digital es que no se da de forma aislada, sino que su naturaleza responde a problemáticas que merecen ser contextualizadas.

La falta de acceso material está claramente relacionado con diferencias socioeconómicas en sociedades específicas. Las deficiencias en acceso a las competencias no pueden ser resueltas sin realizar mejoras en los recursos educativos. El uso factible de los medios digitales en las comunidades locales es difícil de lograr si no está integrado dentro de un enfoque general de la construcción del tejido social en un ambiente específico.(Van Dijk, 2005, p. I84)

De esta manera el enfoque para analizar la brecha digital se construye a partir de la idea de un proceso de superación de etapas que requieren respuestas diferentes y hacen más compleja la discusión sobre las medidas para abordarla.

\section{Ruta metodológica}

El procesamiento de la información que permitía identificar el "desentrañamiento de las ideologías”3 en los textos, hacía preciso plantearse un diseño metodológico que mediante el procesamiento de los documentos permitiera indagar determinados elementos que conforman el análisis lingüístico, conceptual e ideológico de los textos seleccionados en la muestra.

Se elaboraron "preguntas indagatorias precisas" conocidas como macropreguntas, desde las que se pretendía dar respuesta a los principales objetivos perseguidos. Los resultados obtenidos se interpretaron siguiendo las tres etapas propias del ACD: primero, identificando las propiedades formales del texto (etapa descriptiva); seguido de la identificación de la relación entre texto e interacción (etapa interpretativa); y por último, analizando la relación entre interacción y contexto social, teniendo en cuenta los procesos de producción e interpretación, y sus efectos sociales (etapa explicativa).

Plantearse estos interrogantes fue una herramienta indispensable para: a) captar el significado de los acontecimientos o sucesos; b) percibir propiedades y dimensiones que hacen parte de la información registrada en los documentos; y c) desarrollar durante la exploración de datos, observaciones basadas en el análisis teórico y por consiguiente, conseguir pasar de un nivel descriptivo a uno con mayor grado de abstracción.

La metodología se estableció teniendo en cuenta la necesidad de descifrar las formas utilizadas tanto en los documentos institucionales como en la prensa, para legitimar, reproducir y expresar las posturas discursivas que configuran a nivel social determinadas relaciones de dominación, poder y de conflicto de intereses. Este trabajo se adelantó identificando elementos relativos a: la interdiscursividad, las macroestructuras semánticas, la postura discursiva, el análisis de actores discursivos y de sus acciones, el reconocimiento de los significados colectivos más sobresalientes dentro del discurso, la intertextualidad, el orden del discurso; y finalmente, los valores expresivos de los principales términos utilizados.

La muestra utilizada para la observación en el caso de los documentos mediáticos, se seleccionó del conjunto de noticias que contenían el concepto de brecha digital en los periódicos El Mundo y El País, durante el periodo 2000-2009. Estos medios 
constituyen los principales diarios impresos y digitales de circulación en España y cuentan con ediciones digitales que los consagran como medios de referencia en Hispanoamérica.

En el caso de los documentos institucionales, se tomaron cinco documentos que dentro del debate internacional acerca de la inserción de las TIC, han servido de referencia debido al papel relevante de las organizaciones o actores que los promueven: la Declaración de Principios de Ginebra (UIT, 2003), la Agenda de Túnez (UIT, 2005), el informe World Information Society Report (UIT, 2007), el informe Hacia las sociedades del conocimiento (UNESCO, 2005), y el documento Technology for America's economic growth (Al Gore, I993).

Para el análisis de la información se utilizó el programa informático Atlas.ti, el cual brindó el soporte necesario para el procesamiento de los documentos, permitiendo identificar determinados elementos que conforman el análisis lingüístico, conceptual e ideológico de los textos seleccionados en la muestra. Por medio de la metodología propuesta para esta observación se pretendía analizar qué diferencias estructurales y conceptuales de los discursos contribuyen o no a reforzar determinadas referencias acerca del marco axiológico que conforma el paradigma de la sociedad de la información y el de las sociedades del conocimiento en los que se inserta el concepto de brecha digital, en términos de:

\section{Universo đemático}

Por medio del análisis de las unidades conceptuales, recursos y estrategias lingüísticas que son utilizadas en los documentos para reforzar desde el campo cognitivo el universo referencial construido acerca de determinadas unidades temáticas.

\section{Interacción entre los aciores}

Determinar la manera como desde el discurso se legitima el papel de los actores dentro de la estructura organizativa que enmarca el escenario de la sociedad de la información y el de sociedades del conocimiento. Así como identificar el conjunto de estrategias utilizadas por los participantes del discurso para legitimar sus acciones, mitigar los actos de habla, nombrar a otros actores; entre otros mecanismos utilizados mediante el lenguaje para dar forma a las intenciones de los actores discursivos.

\section{Posturas Discursivas}

- Identificar características del discurso que refuerzan determinadas relaciones de poder social dentro del contexto histórico, social, económico y político en que se enmarca el escenario de la llamada sociedad de la información.

- Observar de qué forma los textos analizados se relacionan con textos previos adoptando datos de referencia para reproducir un discurso previo institucional o teórico que hace parte de una tendencia o posición ideológica superior y que por consiguiente, configura una determinada elaboración cognitiva de la información.

- Determinar el conjunto de rasgos lingüísticos y no lingüísticos inherentes al texto, propios del proceso de producción social de los documentos oficiales y de los mediáticos y de la relación de éstos en términos de orden del discurso y prácticas sociales.

\section{Dimensiones del Análisis Propuesto (Modelo Fairclough)}

\section{I).Análisis de las prácticas del discurso}

Comprende aquellos elementos de análisis pertinentes para determinar el contexto social y contexto del texto del que hace parte el discurso (social, político, histórico, psicológico, etc.). Consiste en establecer la variedad discursiva y el tipo de discurso al que pertenece el texto por medio del análisis de la interdiscursividad y la intertextualidad.

- Intertextualidad: En el caso de la presente investigación, en una etapa descriptiva fue interesante observar cómo el lenguaje institucional se transformaba en los documentos mediáticos o viceversa. Para la etapa explicativa 
fue importante analizar también en términos de hegemonía, qué tipo de documentos fuentes prevalecían a través de los diferentes textos emitidos en torno a un tema específico.

- Interdiscursividad: Se indagó tanto en los documentos mediáticos como en los oficiales, las características del género en función de los términos de cómo se ha producido, distribuido y consumido. Tanto en las etapas descriptiva, interpretativa y explicativa fue importante retomar los planteamientos que hace Martín Serrano (2004) sobre las características de producción de medios y la comunicación pública.

- Orden del discurso: En la investigación se prestó atención al discurso dentro del cambio social, con el objetivo de determinar en qué parte el discurso ha incidido en el inicio, desarrollo y consolidación de un cambio. Se observaron dos tendencias que también hacen parte del discurso acerca del capitalismo contemporáneo (Fairclough, 1995):

Mercantilización: Entendida como el proceso mediante el cual los diferentes actores sociales y las instituciones, cuyo interés no es directamente la producción de bienes desde el punto de vista económico, están organizados y conceptualizados en términos de producción, distribución y consumo.

Tecnologización: Propia de la sociedad moderna donde las tecnologías están tomando un carácter de técnicas trans-contextuales, las cuales son mostradas como recursos o herramientas que pueden ser usadas para adoptar una amplia variedad de estrategias en diversos contextos.

\section{2).Análisis del texio}

Esta fase abarca los aspectos formales que dan cuenta de la práctica del discurso. Norman Fairclough (I995) propone tener en cuenta los valores de experiencia, relacionales y expresivos del texto al momento de abordar los diferentes elementos de análisis observados en los documentos. Se trata de características formales que el discurso puede tener.

- Valores expresivos de las palabras: Durante la fase interpretativa fue oportuno responder a cuestiones como: ¿Qué valores experienciales tienen las palabras? - para contestar esta pregunta fue útil alternar la observación entre el texto en sí mismo y el tipo de discurso al que pertenecía, incluyendo esquemas de clasificación de acuerdo a los términos en los cuales el vocabulario fue organizado en el tipo de discurso-, ¿ ‘sobre qué esquemas de clasificación de palabras fueron redactados los textos?, ¿se evidencian palabras con alguna carga ideológica?, ¿qué relaciones de significación relevantes en términos de ideología fueron utilizados?

\section{- Figuras Retóricas:}

a). Metáforas: Las metáforas se valoraron en los términos en que Fairclough lo entiende, como elemento de análisis metodológico. La metáfora es una forma de representar un aspecto de la experiencia en términos de otra, razón por la que interesa ver la relación entre metáforas alternativas que tienen un interés particular en el texto analizado, y entre las metáforas que tienen diferentes componentes ideológicos. La forma como una experiencia vital es metaforizada, es uno de los intereses sobre las luchas y sobre la práctica del discurso.(Fairclough, 1995, p. II9) (la traducción es propia)

b). Metonimias: Con el uso de este recurso se expresan aquellas unidades sobresalientes que proporcionan facilidades nemotécnicas y se posicionan como centrales dentro de una categoría; lo que representa el grupo de objetos y seres del mundo que se ubican conceptualmente como similares por efecto de la generalización o discriminación (Rosch citado en Pardo, 2007).

c). Macroestructuras semánticas: El trabajo realizado con los documentos -en cuanto al acto de determinar los temas que conforman 
metodológicamente los diferentes puntos de análisis y observación-, se realizó a partir de la propuesta de Van Dijk que introduce el concepto de macroestructuras semánticas, para dar cuenta del asunto "de que trata" el discurso a un nivel discursivo, cognitivo y social (Van Dijk, I99o).
En esta etapa se estableció una lista de temas que surgieron a partir de la exploración de los documentos que en una etapa posterior se reagruparon bajo siete grandes macroestructuras semánticas (Tabla I). En una etapa interpretativa y explicativa estas constituyeron la matriz base de la observación que condujo a los resultados de la investigación que se exponen más adelante.

Tabla 1. Macroestructuras Semánticas

TEMA 1: Los planes de desarrollo elaborados por los gobiernos y las demás partes interesadas relacionados con la introducción de las TIC dentro de los diferentes procesos sociales-, deben estar encaminados a garantizar el cumplimiento de los derechos civiles, así como al fortalecimiento de la ciudadanía en lo que respecta al acceso a la información y al conocimiento y los derechos de la comunicación. Este debe ser el eje central sobre el que se fundamente un escenario de transformación tecnológica.

TEMA 2: Se evidencia una falta de seguimiento a los puntos acordados en el Plan de Acción de la CMSI; a algunas líneas del plan se les da mayor importancia que a otras. Es importante hacer un seguimiento adecuado sobre los logros alcanzados en cada país en cuanto a los diferentes aspectos que conforman el escenario propuesto de sociedad de la información.

TEMA 3: Los indicadores utilizados para medir el grado de adaptación de un país al modelo planteado por la sociedad de la información, responden principalmente a cuestiones relacionadas con el grado de acceso a las herramientas tecnológicas; dejando de lado la implementación de sistemas de medición capaces de resolver cuestiones como el tipo de uso, desarrollo de capacidades, nivel de aprove- chamiento u otros problemas estructurales que aún no se han superado ni evaluado adecuadamente.

TEMA 4: Posicionar la discusión en torno a los temas que integran el escenario de sociedades del conocimiento permite entender la complejidad de ciertos elementos que van más allá de la discusión sobre la brecha digital y la sociedad de la información. Esto hace posible analizar qué tipo de sociedad se está estructurando con la implementación de las TIC dentro de los diferentes procesos sociales.

TEMA 5: El acceso a la información y al conocimiento por medio de las herramientas TIC no puede quedar en manos de la lógica general de producción y circulación de mercancías.

TEMA 6: Se legitima el poder intrínseco de las TIC y de internet dentro de procesos como la comunicación o demás prácticas sociales, bajo promesas de desarrollo social; desconociendo la necesidad de contemplar transformaciones sociales de tipo estructural.

TEMA 7: Los intereses comerciales y políticos detrás de las iniciativas relacionadas con la introducción de las TIC dentro de los diversos procesos sociales ponen en riesgo que se cumplan los diferentes objetivos sociales esperados en un escenario de sociedad de la información y de sociedades del conocimiento.

Fuente: Elaboración Propia 
- Lo que se omite en el texto:

Al tiempo que se reconocían las temáticas que conformaban el mapa referencial con el que se construye el concepto de brecha digital, la observación permitía identificar aquellas temáticas que se pasan por alto o se silencian y que determinan también el tipo de información que se elabora respecto a un asunto.

d). Análisis de los Actores y sus Acciones: Teniendo en cuenta que las acciones permiten visualizar la implicación directa de los actores y dentro del sistema social aluden a las interacciones propias de la sociedad, Neyla Pardo remite a la figura del actor discursivo trabajado por Bajtin, la cual permite entender el carácter dialógico constructor del discurso y plurisignificativo del discurso, en la medida en que es a través de este y de sus voces que se desentraña la acción comunicativa (Pardo, 2007, p. 93).

Este tipo de actor se diferencia de los otros porque es el participante activo de la interacción, desempeña roles discursivos y construye como sujeto social una imagen de sí mismo, del otro y de la realidad. Por esta razón en la presente investigación la observación realizada se centró en el análisis de los actores discursivos y de sus acciones presentes en el discurso, también teniendo en cuenta la manera como se concibe a los sujetos dentro del modelo canónico de la mediación cognitiva propuesto desde la Teoría de la comunicación.

\section{3). Análisis de la práctica sociald de la cual el discurso hace parte}

Con el objetivo de especificar la naturaleza de la práctica social de la cual el discurso hace parte, que es la base para explicar la naturaleza que determina la práctica del discurso y por consiguiente los efectos que dicha práctica tiene en un sistema social, Fairclough propone indagar sobre los procesos socioculturales de los que hace parte el texto, así como también acerca de sus probables efectos. Con ello se busca obtener información relevante acerca de: a) las condiciones sociales que delimitan el discurso (incluyendo las económicas y políticas); b) sus efectos sociales en términos de sistema de conocimiento y creencias (e ideologías); c) relaciones sociales de poder; y d) el papel de los actores como sujetos sociales. Esto también da cuenta de los cambios sociales y culturales.

Este análisis de la práctica también fue de gran utilidad para analizar las diferentes representaciones sociales creadas en el discurso por medio de la observación del sistema de conocimientos y creencias, las relaciones sociales y las identidades sociales que se crean en el discurso a propósito de temas relacionados con los temas y subtemas que conforman los enfoques de sociedad de la información y sociedad del conocimiento. Este trabajo permitió en una fase posterior, desentrañar la postura discursiva que se sostiene por medio del uso de diferentes estrategias lingüísticas. Este proceso resultó ser definitivo para finalmente llegar a conclusiones sobre la práctica social del mismo.

Tabla 2. Categorización de los actores discursivos dentro de un contexto comunicativo

\begin{tabular}{lll}
\hline Contexto Comunicativo & Actores Discursivos & \multicolumn{1}{c}{ Actores Sociales } \\
\hline De intervención & Divulgadores & $\begin{array}{l}\text { a. Ejecutor - Promotor, b. Informante - divulgador, } \\
\text { c. Observador - Evaluador }\end{array}$ \\
\hline Promotores & \begin{tabular}{l} 
a. Ejecutor - Promotor, b. Observador - Evaluador \\
\hline Controladores
\end{tabular} & \begin{tabular}{l} 
a. Observador - Evaluador, b. Regulador, c. Ejecutor - Promotor \\
\hline De recepción
\end{tabular} \\
\hline Críticos & $\begin{array}{l}\text { a. Observador - Evaluador, b. Informante - divulgador, } \\
\text { c. Ejecutor - Promotor, d. Regulador }\end{array}$ \\
\hline
\end{tabular}

Fuente: Elaboración Propia 


\section{Hallazgos}

a). Los objetivos que se persiguen para lograr un modelo de sociedad de la información están determinados por un modelo socioeconómico donde la fuerza del mercado, determina la inserción del cambio tecnológico.

La capacidad de los países para adaptarse a un nuevo escenario depende de su capacidad para difundir infraestructura tecnológica y por consiguiente, demostrar mayores indicadores de crecimiento económico. Es evidente un reduccionismo al hecho tecnológico motivado específicamente por el crecimiento económico, donde las redes de producción de la información determinan el lugar de una sociedad.

Sobresale la importante proporción concedida a referencias en torno al tema de las TIC. Esto se relaciona especialmente desde el imaginario respecto al ritmo tecnológico que se debe seguir para no quedar relegado del tren del progreso. Así, se presentan y justifican las iniciativas de inversión tecnológica, la adaptación del marco legal que contribuye a su consolidación y la participación de actores con capacidad de acción para hacer posible esta transformación.

La innovación técnica y las nuevas relaciones sociales están presentes en la política de reindustrialización que conforma el discurso político actual. Los países considerados desarrollados, diseñan sus políticas sobre el discurso de la modernización y exhortan a sus vecinos a repetir sus modelos económicos con la promesa de salir de un estado de subdesarrollo. Bajo esta estructura las relaciones de dependencia tecnológica se perpetúan y generan nuevas formas de dominación económica y cultural.

El rol discursivo que ejercen los representantes del sector privado como ejecutores/promotores de iniciativas de tipo comercial, o ejerciendo un papel activo dentro de las consideraciones derivadas de las reuniones de trabajo en las cumbres, es estratégico. También ejercen un rol de controladores, reivindicando sus derechos comerciales como pro- ductores de conocimientos o elaborando informes donde participan en el diagnóstico de temas, como la infraestructura tecnológica necesaria para superar la brecha digital. De esta manera se consolida su papel como operadores de servicios tecnológicos a nivel trasnacional, desde donde controlan toda la cadena de información, acceso a la conexión y producción de contenidos.

La postura discursiva legitimada desde algunos documentos oficiales que favorece el papel relevante del sector privado, se reproduce en los medios a través del uso de estrategias lingüísticas que remiten al simbolismo colectivo propio del uso de los denominados tópicos. En este caso el recurso al tópico de ventaja-utilidad, permite legitimar la importancia de la convergencia de medios, de plataformas abiertas y de mayor dimensión al mercado, para que los ciudadanos tengan garantizados los servicios de la sociedad de la información.

A través de estrategias discursivas como la mitigación, se reduce el papel de este agente y de sus intereses comerciales, utilizando expresiones del tipo "coalición de grandes socios" para reemplazar términos que pueden resultar controvertidos como: participación del sector privado, intereses de empresas de software, etc. De esta forma se presenta como algo natural la alianza e intervención del sector privado.

Asimismo el concepto de libre mercado se promociona de la mano del consumo de bienes materiales y culturales, de los que no escapa la tecnología de la información. De la misma manera, las demandas de tipo comercial en el campo de acceso a las nuevas tecnologías son utilizadas dentro del discurso por medio de estrategias discursivas como el uso del tópico de justicia, donde se reclama una igualdad de acceso a los servicios info-comunicacionales para todos los ciudadanos.

Todo esto ocurre dentro de un orden social caracterizado por una fuerte mercantilización en sus procesos estructurales, de los que la generación de conocimiento no escapa; por ejemplo en el caso de los procesos de aprendizaje, los cuales se retoman a partir de la introducción de expresiones relacionadas con las actividades productivas. 
Dichos procesos socio-económicos determinan la comercialización del conocimiento y no su intercambio, teniendo en cuenta que la información y el conocimiento hacen parte de las reglas de producción y circulación de mercancías. De esta manera surgen nuevas formas de exclusión, propiciadas por la fuerte concentración de poder en manos de los países industrializados y de las grandes empresas transnacionales.

Toma sentido analizar si más allá del debate sobre la necesidad de elegir un escenario de sociedad de la información o uno de sociedad del conocimiento, es necesario detenerse a reflexionar de qué manera las redes de producción de la información determinan el lugar de una sociedad.

b). El modelo de sociedad de la información planteado desde el discurso institucional público se caracteriza por ser presentado como un proceso lineal y uniforme a nivel global, donde las diferencias culturales y estructurales de los diferentes grupos sociales pierden cada vez más la oportunidad de ser valoradas.

La forma como se construye la referencia en torno a este tipo de sociedad incide en que se contemple una única sociedad de la información, un fenómeno que para Hamelink (citado en UNRIDSD, 2003) significa ignorar que las sociedades enfrentan avances informacionales de muchas maneras diferentes, a distintas velocidades y en contextos históricos diferentes. Mientras que desde un enfoque de sociedades del conocimiento tal como lo concibe la UNESCO, se contemplan las diferentes transformaciones sociales desde una perspectiva de diversidades culturales, donde prima el valor de la heterogeneidad y pluralidad propios de una visión que legitima la diversidad de conocimientos.

La expresión misma de sociedad de la información en su conformación, antepone el concepto de información sobre el de comunicación, ocasionando una falta de análisis crítico sobre el papel de la comunicación en la reproducción y sustentabilidad de los procesos sociales, culturales y políticos. c). El carácter homogenizador del concepto de brecha digital contribuye a legitimar el acceso a la tecnología como solución única y adecuada para que los individuos consigan un real aprovechamiento de las TIC y sean capaces de procesar $y$ generar conocimiento.

El concepto se mantiene en un discurso abstracto, propio de la inserción de un término que no se ha terminado de definir y que apenas se está constituyendo dentro de un marco referencial y cognitivo. Su construcción aparece a partir de la inclusión de determinados temas relacionados con el sobredimensionamiento del poder de la tecnología como herramienta generadora de cambio; y al tiempo de la exclusión de ciertos temas relacionados con las fases de motivación, conocimientos y usos avanzados; los cuales conforman un proceso donde deben ser superadas diversas etapas para lograr así una real transformación y aprovechamiento de los dispositivos tecnológicos. Este carácter homogenizador del concepto de brecha digital se legitima en los documentos en una suerte de metonimia de la parte por el todo donde la brecha de tipo digital se utiliza para definir diferentes problemáticas que responden mayormente a temas relacionados con una brecha de tipo cognitivo.

El universo referencial de esta brecha está conformado por elementos que implican un orden relacional de: acceso y consumo de bienes como garantía para estar desarrollados, evitar quedar así por fuera de las redes de producción y por consiguiente, hacer parte de un escenario de sociedad de la información. Este es un discurso que responde a ideales neoliberales de consumo de herramientas tecnológicas como único medio y garantía para evitar ser excluidos.

Tal como lo describe Cheikh Guèye (citado en UNRISD, 2003), el término brecha digital en su expresión francesa-la fracture numérique-implica que con anterioridad existió una totalidad, es decir una supuesta igualdad tecnológica anterior o cierta totalidad mundial a la cual deberíamos aspirar dentro de un modelo de sociedad única 
de la información. De la misma manera, la idea de fractura o abismo contribuye a reforzar el imaginario sobre la brecha a partir de situaciones que se enfrentan: los que tienen acceso y los que no, y no como un proceso de diferentes situaciones que se deben abordar desde una perspectiva de cambio continuo.

El estado de brecha también es visto como un acontecimiento con naturaleza propia que deja de ser abordado como un hecho psicosocial para remitirse a un hecho físico-biológico donde las reacciones para tratarlo son vistas bajo principios naturales e incuestionables, y no bajo un enfoque de diferenciaciones digitales en donde se reconoce el proceso constante de aprendizaje y de generación de conocimiento para saber interactuar ante las nuevas aplicaciones tecnológicas que van surgiendo.

Desde el discurso se desconoce que la brecha responde a un proceso dinámico, cambiante y complejo, con múltiples factores internos y externos que intervienen en el mismo. Estos factores responden a problemas estructurales de fondo que necesitan a la vez ser resueltos desde diferentes realidades y no solo desde las tecnologías. De esta forma, parece oportuno hablar de brechas que aparecen continuamente con la evolución de la tecnología, los modos de acceso y otros factores propios de un entorno cambiante.

d). La implementación de las TIC viene acompañada de un discurso que concentra virtudes intrinsecas en el papel de las herramientas, sin tener en cuenta que el acceso a las herramientas, es tan solo una de las dimensiones que deben ser valoradas.

Esta relación que desde el discurso se reproduce entre las tecnologías y el desarrollo, se legitima por medio del uso de expresiones que refuerzan el potencial de las tecnologías como una fuerza capaz por sí sola de generar cambios necesarios en términos de progreso y desarrollo.

La construcción de la representación en torno a la inserción de las herramientas tecnológicas como un fin en sí mismo se hace evidente, esta sin considerar que se trata tan solo de un medio que, acompañado de una serie de factores, puede facilitar un proceso. El poder asignado a las TIC se relaciona con el logro de objetivos de desarrollo, mientras que la conectividad se presenta como garantía para asegurar el progreso de un país y el bienestar de sus habitantes.

Esta capacidad atribuida a las tecnologías se inserta dentro de un marco referencial relacionado con temas de desarrollo y superación de problemas como la brecha digital para la cual se proponen iniciativas relacionadas específicamente con el acceso material a los dispositivos. Por consiguiente, se acentúa un pensamiento de determinismo tecnológico, bajo el cual las tecnologías se encuentran por encima de las decisiones que puedan tomar los ciudadanos, en términos de garantizar un enfoque de derechos civiles.

Los postulados del documento Technology for America's economic growth han sido retomados por los medios de comunicación para reforzar la idea de que la inversión en infraestructura tecnológica asumida por muchos países desde la década de I99o ha sido indispensable en términos de competitividad para alcanzar mayores niveles de desarrollo. Dentro del lenguaje de la tecnologización se favorece la idea de que el cambio tecnológico es un hecho de evolución global de la que ningún país puede escaparse.

En este mismo documento es interesante observar la forma como se atribuye a la tecnología el poder de multiplicar el potencial para la innovación y el progreso. Esta postura ha incidido en otros documentos institucionales y noticias en las que el poder de las tecnologías aparece en medio de un lenguaje en el que los verbos y calificativos con los que se construye la imagen del objeto de referencia determinan el poder intrínseco que se les asigna dentro de los diferentes procesos sociales. Todo esto se da bajo una visión eminentemente positiva, donde queda descartado cualquier escenario de riesgo que pueda generar el proceso de transformación propiciado por las nuevas tecnologías. 
Dentro de esta tendencia se dejan en un segundo plano las repercusiones relacionadas con la comunicación, específicamente con promover el diálogo entre las personas. En cambio, se contribuye a mantener la continuidad de un modelo socioeconómico bajo el cual los diferentes actores justifican la inversión en equipamiento tecnológico amparados bajo las promesas de competitividad propias de la nueva economía global.

Se identificó asimismo la tendencia a legitimar determinadas iniciativas a partir de experiencias anecdóticas con las que se pretende ejemplificar un caso significativo, pero que no se traduce en indicadores que puedan medir las repercusiones de las TIC con un enfoque metodológico apropiado.

e). Se pasa por alto el tema de la generación del conocimiento como requisito indispensable para la transformación propuesta, dando mayor valor a otras consideraciones de tipo económico, tecnológico o político.

Es notable el poco reconocimiento que se hace desde los documentos a analizar en temas como la gestión del conocimiento que circula por la red o a la que tienen acceso los individuos. Exceptuando el caso de la UNESCO, la propuesta de potenciar el aprovechamiento compartido del conocimiento no tiene ningún eco en los documentos y mucho menos en los medios de comunicación. Hacer la diferenciación en el discurso, entre una brecha de tipo digital y otra de tipo cognitivo, es trascendental para que las iniciativas tengan en cuenta respuestas de tipo técnico y al tiempo, aplicaciones para el fomento de la enseñanza y generación de conocimiento que garanticen una real transformación de los procesos de cambio social.

En cuanto a la construcción de la referencia en torno al grado de aprovechamiento de una herramienta tecnológica, el caso particular de la alfabetización digital se suele construir sin hacer distinción entre las habilidades impartidas con la simple transmisión de conocimientos de ofimática tradicional, y el hecho de brindar los mecanismos para que los usuarios sepan apropiarse de las herramientas. Se deja de lado el fomento de los verdaderos procesos de conocimiento, relacionados especialmente con la capacidad de aprender a aprender; y el hecho de reconocer que las literacidades digitales son múltiples y condicionadas por las circunstancias particulares de los individuos (condiciones socioeconómicas, capacidades cognitivas, etc.).

En el caso concreto de la aplicación de las TIC dentro del ámbito educativo, el mayor número de referencias encontradas relacionadas con este tema están vinculadas totalmente con las aplicaciones concretas de las nuevas tecnologías que se hacen dentro de los procesos de aprendizaje. No se encontraron menciones relacionadas con temas como la adaptación de los planes de estudio o sobre las implicaciones de esta modernización pedagógica.

f). La falta de mayor relevancia a tratar temas relacionados con el aporte que pueden prestar las TIC para desarrollar procesos más participativos, propios de un contexto de sociedades del conocimiento es evidente.

Estos procesos son fundamentales para que los mismos ciudadanos sean quienes produzcan contenidos, donde se fortalezcan nuevos instrumentos de organización social y se garantice la participación ciudadana en las decisiones que se toman y que les afectan como colectivo. Se pasa por alto que el verdadero fin del cambio tecnológico debe ser adelantar el camino hacia el desarrollo humano dentro de un marco referencial de derechos y que las tecnologías deben estar al servicio de las necesidades particulares de las comunidades.

La representación social de las personas-ciudadanos está determinada por una tecnologización en el orden del discurso, donde los individuos son denominados como internautas: usuarios de una herramienta y no como usuarios-ciudadanos capacitados para desarrollar competencias cognitivas y estar empoderados para participar e intervenir en las decisiones propias de un entorno de sociedades del conocimiento. 
La falta de participación del actor que suponen las organizaciones internacionales, como observador o evaluador de los procesos sociales influye en el hecho de que su aporte en el debate por las garantías en derechos como la libertad de expresión y los principios democráticos, no se muestre tan marcada dentro del escenario social. Sería interesante que su intervención también se diera en términos de participar como reguladores que contribuyan con sus acciones a evaluar las diferentes iniciativas emprendidas bajo estrategias comerciales o políticas y de esta forma ser garantes de que cumplan las necesidades reales de los ciudadanos.

Respecto a asuntos directamente relacionados con derechos y garantías sociales, se observó la presencia de expresiones que sirven para legitimar la necesidad de implementar medidas de control y vigilancia para defender los intereses de los ciudadanos, generando por consiguiente un incremento en la implantación de sistemas de vigilancia, de rastreo de datos y del comportamiento individual ante los que los usuarios no son capaces de reaccionar para defender sus derechos individuales frente a las instituciones.

g). La naturaleza misma de las organizaciones que formulan las recomendaciones a través de sus documentos determina el tipo de soluciones propuestas para superar problemas como la brecha digital.

Es notable la capacidad que se le atribuye en estos documentos a las TIC como garantía para producir grandes transformaciones en varios aspectos de la vida de los habitantes, así como para legitimar el imaginario sobre la necesidad de respaldar políticas de inversión en infraestructura tecnológica, como requisito indispensable para hacer parte de la sociedad de la información y alcanzar mayores niveles de desarrollo. Estas recomendaciones corresponden a los objetivos y campos de acción de las organizaciones. Organizaciones como la UIT de carácter técnico, proponen soluciones de acceso a tecnologías más fáciles y ase- quibles, mientras que agencias como la UNESCO reconocen la existencia de una brecha ante todo de tipo cognitivo, que debe ser entendida dentro de una dinámica de diferencia de conocimientos y de optimización de las capacidades cognitivas de los habitantes.

Desde los documentos institucionales (especialmente los de la CMSI y la UIT) se relaciona el poder de las TIC con el logro de objetivos de desarrollo, impulsando ideas tales como que la conectividad va a garantizar el progreso de un país y el bienestar de sus habitantes. Además se refuerza el imaginario de superación de la pobreza gracias a la inserción de las tecnologías, dejando de lado una cuestión fundamental que Hernández Muñoz (2008) señala como un punto de análisis trascendental: ¿Cómo hacer que esa transición sea eficiente y equitativa en países que son, estructuralmente, inequitativos y de baja eficiencia relativa?

En el caso específico de la brecha digital las medidas planteadas desde los documentos institucionales y retomadas en los medios, pasan también por priorizar los objetivos de tipo económico mediante la adaptación de las nuevas tecnologías; esto en la mayoría de los casos sin contemplar que la brecha hace parte del conjunto de inequidades que las relaciones de poder propician al interior mismo de las sociedades. De esta manera, desde el discurso se desconoce que la disminución de esta brecha solo puede entenderse en el contexto de unos cambios mucho más amplios.

\section{h). Los documentos analizados, bajo determinadas estructuras lingüisticas, contribuyen a la legiti- mación de un marco regulatorio caracterizado por un entorno de liberalización, privatización y competencia para conseguir logros en la adap- tación de las transformaciones tecnológicas.}

A partir de la presencia de un vocabulario que hace referencia a un modelo de desarrollo tecnológico cuyo fin último es el crecimiento económico, se legitiman las propuestas de competitividad, desregulación y privatización de los servicios que hacen parte del marco en el que se propone 
que deben insertarse las TIC para alcanzar un desarrollo de tipo económico. Este argumento opera en oposición a la idea de una falta de rendimiento o estancamiento económico que tanto se teme, pero que no necesariamente se relaciona con un tipo de desarrollo sostenible.

Se favorece la función de la empresa privada presentando sus iniciativas o resaltando la utilidad de una acción emprendida desde el sector privado, afianzando de esta manera el mercado tecnológico. Se insta a los gobiernos a generar un entorno favorable para que se atraiga la inversión privada y así cumplir con el objetivo de desarrollar la infraestructura TIC necesaria para garantizar las demandas de todos los ciudadanos que necesitan acceder a los servicios tecnológicos.

En medio de un modelo social que atribuye fuerte valor a la gestión de los agentes privados, se legitima desde el discurso la gestión de este actor a través del uso de metáforas como la libertad, cuyos componentes ideológicos remiten a la doctrina del free flow of information, que se muestra como la única vía posible para que los ciudadanos tengan garantizados sus derechos a acceder a los servicios ofrecidos por las nuevas tecnologías.

La ausencia de actores categorizados como expertos legitima la necesidad de la intervención de un marco regulatorio marcado por la desregularización y la competencia, también impide que se genere un debate social acerca de la necesidad de políticas de acceso universal o de un modelo alternativo que permita que todos los ciudadanos tengan acceso a las tecnologías sin estar limitados por su capacidad de pago.

Se hace evidente la utilización de un lenguaje propio de la gestión empresarial -promovido especialmente por actores representantes del ámbito público-, rico en expresiones que se ajustan a un modelo socio-económico y al entorno habilitador propio de la nueva economía global. Se puede identificar asimismo el uso de un lenguaje propio del mapa referencial con el que también se ha construido el discurso sobre el modelo económico neoliberal.

Este ejercicio de análisis crítico del discurso constituye un ejercicio oportuno para reforzar el debate sobre la universalización democrática del saber y del conocimiento, en donde muchos mitos que prometen igualdad y democracia por el uso de las nuevas tecnologías deben ser reemplazados por escenarios reales donde los procesos culturales tengan en cuenta las necesidades de acceso al saber y al conocimiento de todos los ciudadanos.

Asumir una posición crítica frente al tipo de discurso con el que se legitima la inserción social de las nuevas tecnologías permitirá desarrollar una real apropiación social de estas herramientas, teniendo en cuenta los intereses colectivos de los usuarios y aprovechando las potencialidades tecnológicas. También será un mecanismo indispensable para lograr que las políticas de comunicación partan de un real consenso, donde la opinión de los ciudadanos juegue un papel protagónico en su planeación e implementación.

\section{Referencias}

Barbero, J. M. (2008, abril). Diversidad cultural y convergencia digital. Conferencia dictada en la Facultad de Ciencias de la Información de la Universidad Complutense de Madrid, España.

Fairclough, N. (1995). Media Discourse. England: Arnold Ed.
Felicie, A. (2006). Biblioteca pública, sociedad de la información y brecha digital. Buenos Aires: Alfagrama Ediciones.

Gore, A. (I993). Technology for America's Economic Growth, A New Direction to Build Economic Strength. Vice President Albert Gore, Jr. Recuperado de http://www.mcreporter.info/ documenti/technology.pdf 
Hernández, E. (2008, noviembre). Hacia las comunidades de conocimientos. En XIII Congreso Internacional del CLAD sobre la Reforma del Estado y de la Administración Pública, Buenos Aires, Argentina.

Marí, V y Sierra, F. (2008, enero). Capital Informacional y apropiación social de las nuevas tecnologías. Las redes críticas de empoderamiento local en la Sociedad Europea de la Información. Revista Telos. Recuperado de http://sociedadinformacion.fundacion. telefonica.com/telos/articulodocumento.asp@ idarticulo $=2 \& \mathrm{rev}=74 \cdot \mathrm{htm}$

Martín Serrano, M. (2004). La producción social de la comunicación. Ediciones i y 3. Madrid: Alianza Editorial.

Martín Serrano, M., Piñuel, J. L, Gracia, J. y Arias, M. (I982). Teoría de la comunicación. I: Epistemología y análisis de la referencia. Madrid: Corazón Editor.

Mattelart, A, Mattelart, M. y Multigner, G. (I988). Pensar sobre los medios. Comunicación y crítica social. San José de Costa Rica: Editorial Departamento Ecuménico de Investigaciones DEI.

Pardo, N. (2007). Cómo hacer análisis crítico del discurso. Una perspectiva latinoamericana. Chile: Editorial Frasis.

UNESCO. (2005).Hacia las sociedades del conocimiento. Informe Mundial de la UNESCO.
UIT (Unión Internacional de Telecomunicaciones). (2003). Declaración de Principios. Cumbre mundial sobre la sociedad de la Información. Recuperado de http://www.itu.int/ wsis/index-es.html

UIT (Unión Internacional de Telecomunicaciones).(2005). Agenda de Túnez para la sociedad de la información-Cumbre Mundial para la sociedad de la Información. Recuperado de http://www.itu.int/wsis/indexes.html

UIT (Unión Internacional de Telecomunicaciones).(2007). World Information Society Report 2007 .

UNRISD. (2003). Análisis de los avances informacionales: Reflexiones sobre temas esenciales de investigación. Recuperado de http://www. unrisd.org/80256B $3 \mathrm{Coo}_{5} \mathrm{BCCF}_{9} /$ (httpPubli

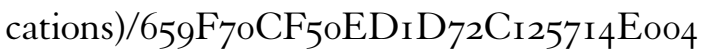
B9DEF? OpenDocument

Van Dijk, J. (2005). The deepening divide. Inequality in the Information Society.London: Sage Publications.

Van Dijk, T. (1990). La noticia como discurso: comprensión, estructura y producción de la información. España: Paidós Comunicación

Van Dijk, T. (200o). El discurso como estructura y proceso. Barcelona: Editorial Gedisa.

Vega-Almeida, R. (2007, abril). Brecha digital: un problema multidimensional de la sociedad emergente. Revista Inclusão Social, (2), 96-io8.

Notas

1. Para esta investigación el trabajo realizado por Manuel Martín Serrano con su Teoría de la mediación comunicativa ha sido un eje fundamental. Por esta razón tomamos del autor el concepto que utiliza en sus modelos de objetos de referencia, para designar los objetos materiales o ideales que asociados, bajo expresiones o nominaciones, generan las diferentes representaciones que hacen parte del proceso comunicativo.

2. El Profesor tomaba como ejemplo para explicar esta afirmación el caso de la población desplazada en Colombia, que en un alto porcentaje tiene acceso al teléfono móvil, "empezamos a quedarnos en la brecha digital y no nos enteramos que la inmensa mayoría de los millones de desplazados en Colombia tienen móvil".

3. Expresión utilizada por Neyla Pardo al referirse a la identificación de las ideologías presentes en el discurso. 
